\title{
Novel Unipolar Sign Encoded OFDM for Next Generation PONs
}

\author{
Mohammed M. Mohammed ${ }^{1}$, Abdulaziz E. El-Fiqi ${ }^{2}$, and Ziad A. El-Sahn' \\ ${ }^{I}$ Photonics Group, Electrical Engineering Department, Alexandria University, Alexandria 21544, Egypt \\ ${ }^{2}$ Egypt-Japan University of Science and Technology (E-JUST), Alexandria 21934, Egypt \\ alsarariomhammed@gmail.com,abdulaziz.elfiqi@ejust.edu.eg,ziad.elsahn@ieee.org
}

\begin{abstract}
We propose a novel unipolar encoding technique for orthogonal frequency-division multiplexing (OFDM) that increases the bandwidth efficiency by $33 \%$ and reduces computational complexity by $50 \%$ compared to conventional intensity modulation/direct detection (IM/DD) OFDM systems.

OCIS codes: (060.2330) Fiber optics communications; (060.4080) Modulation.
\end{abstract}

\section{Introduction}

Fiber-to-the-home (FTTH) based on a passive optical network (PON) infrastructure is recognized as a future-proof solution that alleviates the increasing bandwidth demand in the access while offering ultra-fast connections to end users. From the other side, orthogonal frequency-division multiple-access (OFDMA) is considered a good candidate for next generation PONs aiming at more than $1 \mathrm{~Gb} / \mathrm{s}$ per user, because of its high spectral efficiency, resilience to inter-symbol interference (ISI), and simple equalization techniques [1,2].

To preserve reduced cost and complexity of a PON, OFDM needs to be slightly modified to work with simple intensity modulation/direct detection (IM/DD) optoelectronics [2]. This puts constraints on the OFDM signal to be real and positive in order to modulate the intensity of light. Several systems have been proposed in that regard, such as DC biased optical OFDM (DCO-OFDM), asymmetrically clipped optical OFDM (ACO-OFDM), and unipolar OFDM (U-OFDM) [3], [4].

In this paper a novel coding technique is applied to the time domain signal after the inverse fast Fourier transform (IFFT) to obtain a real positive signal, instead of applying Hermitian symmetry before the IFFT. This improves the bandwidth efficiency and reduces the complexity compared to previous solutions.

\section{Proposed Unipolar Sign Encoded (USE) OFDM for Next Generation PONs}

In conventional systems, Hermitian symmetry property is used to get a real signal from the IFFT output by adding the conjugate of the complex signal before the IFFT. This doubles the size of IFFT/FFT, and therefore doubles the system complexity. Large FFT sizes require higher bit precision and therefore consume more power and occupy larger chip areas [5]. The proposed USE-OFDM avoids the use of Hermitian symmetry, therefore reducing the complexity and saving bandwidth compared to conventional U-OFDM and ACO-OFDM systems. In terms of bandwidth efficiency, in order to send $N$ symbols we only need $3 N$ samples (explained later) compared to $4 N$ samples for conventional U-OFDM and ACO-OFDM. This means that U-OFDM uses only $25 \%$ of the available bandwidth, whereas the USE-OFDM exploits $33.3 \%$ of the system bandwidth. This corresponds to a $33 \%$ increase in bandwidth efficiency for the proposed USE-OFDM.

In USE-OFDM, after M-quadrature amplitude modulation (M-QAM) data mapping and serial to parallel (S/P) conversion, the signal is passed to the IFFT block as shown in Fig. 1(a). The IFFT complex output is then split into two signals; real part and imaginary part as illustrated in Fig. 1(b). After that, the sign encoder generates a unipolar
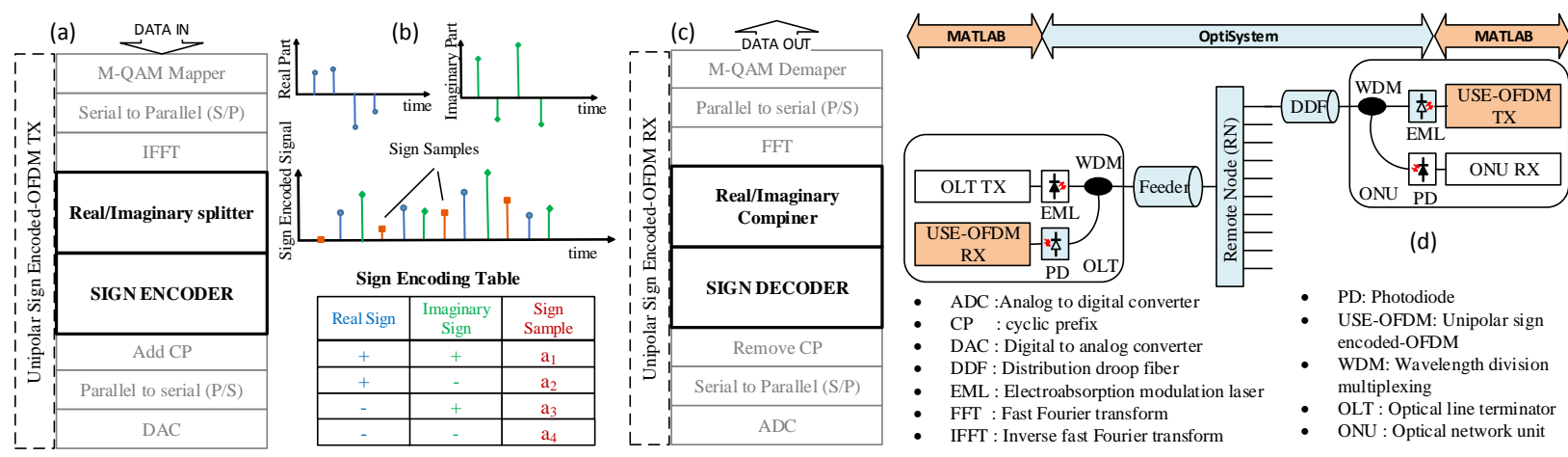

Fig. 1. The proposed USE-OFDM: (a) transmitter structure, (b) illustration of sign encoding, (c) receiver structure, and (d) PON architecture with USE-OFDM in uplink direction (also used as a simulation setup). 
signal by adding a sign sample to each pair of real and imaginary samples. The added sign sample represents the sign of the two parts according to the sign encoding table in the same figure. The sign samples are then added to the signal, while the signs of the real and imaginary parts are removed as per Fig. 1(b). At the receiver side in Fig. 1(c), the unipolar signal is converted back to a complex signal through a sign decoder. This decoder compares the first sample of each three samples with predefined values and chooses the nearest value. Depending on that it then assigns the signs to the next pair of samples, i.e., the real and imaginary parts of the OFDM symbol. The complex signal can then be regenerated when removing the sign samples and passed to the FFT block to recover the data.

In the PON uplink configuration in Fig. 1(d); the USE-OFDM transmitter generates an OFDM signal that directly modulates the intensity of the electro-absorption modulated laser (EML). The signal is then sent over the DDF, RN and through the feeder to the OLT. At the OLT, after photodetection, the signal is fed into the USEOFDM receiver similar to that in Fig. 1(c).

\section{Simulation Setup and Results}

The USE-OFDM signal with 512 FFT length is generated offline using Matlab. The signal is then passed to Optisystem to directly modulate a $1270 \mathrm{~nm}$ EML (compatible with XG-PON) that outputs $5 \mathrm{dBm}$ of power. The signal is then sent over the uplink PON channel in Fig. 1(d). At the receiver side, after photodetection, the signal is sent back to Matlab for decoding and BER calculation. The BER performance of the USE-OFDM versus U-OFDM at 1.25 Gbaud is shown in Fig. 2(a). The two systems have almost the same performance near and below the FEC limit $\left(\right.$ BER $\left.=10^{-3}\right)$. However USE-OFDM outperforms U-OFDM in both system complexity and bandwidth efficiency as explained earlier. As expected, there is performance degradation for both systems for higher M-QAM orders. Fig. 2(b) shows the bit rate contour plots versus the ONU launch power and PON reach at the FEC limit for 32 ONUs. Appropriate modulation order and bitrate could be selected depending on the available launch power and required PON reach. For example at $6 \mathrm{dBm}$ of launch power and for a PON reach of at more than $30 \mathrm{~km}, 4-\mathrm{QAM}$ at $5 \mathrm{~Gb} / \mathrm{s}$ would be preferred over the 5Gb/s 16-QAM. Also for a $20 \mathrm{~km}$ PON reach, 4-QAM and 16-QAM can achieve $10 \mathrm{~Gb} / \mathrm{s}$ with a slight difference $(\sim 1 \mathrm{~dB})$ in launch power.
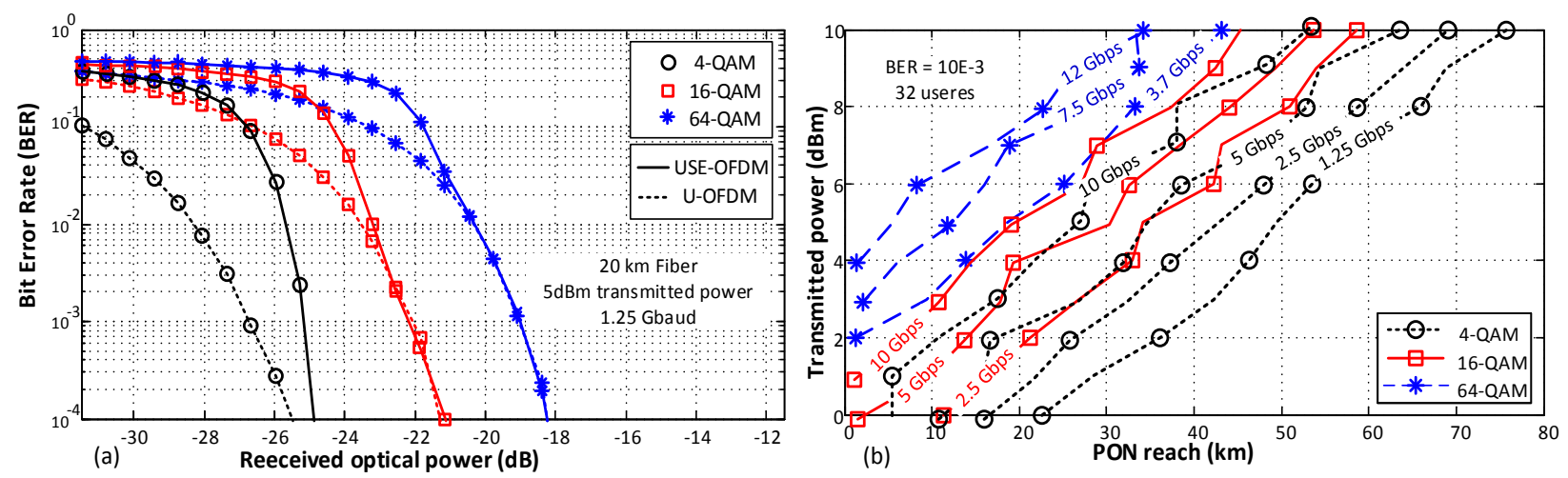

Fig. 2. (a) Uplink BER performance over a $20 \mathrm{~km}$ PON for USE-OFDM and U-OFDM, (b) USE-OFDM at FEC limit and 32 users with different aggregate bit rates.

\section{Conclusion}

A novel approach that increases the bandwidth efficiency and minimizes the complexity of IM/DD OFDM is presented in this paper. This is based on a new coding technique that generates a real and positive OFDM signal appropriate for IM/DD optical communication systems. The performance of the proposed USE-OFDM is similar to that of conventional U-OFDM. However, the proposed system surpasses the conventional U-OFDM system in terms of complexity (50\% savings) and bandwidth efficiency (33\% savings). This gives the proposed system an advantage over conventional U-OFDM and ACO-OFDM systems.

\section{References}

[1] Neda Cvijetic, "OFDM for next-generation optical access networks," J. Lightwave Technology, vol. 30, no. 4, February, 2012.

[2] J. Armstrong, "OFDM for optical communications," J. Lightwave Technology, vol. 27, no. 3, pp. 189-204, February, 2009.

[3] D. Tsonev, S. Sinanovi'c, and H. Haas, "Novel unipolar urthogonal frequency division multiplexing (U-OFDM) for optical wireless," in Proc. Vehicular Technology Conference (VTC Spring), pp. 1-5, Yokohama, Japan, May 2012.

[4] J. Armstrong, and B. J. C. Schmidt, "Comparison of asymmetrically clipped optical OFDM and DC-Biased optical OFDM in AWGN," IEEE Communications Letters, vol. 12, no. 5, pp. 343-345, May 2008.

[5] Fatima Barrami, et al., "A novel FFT/IFFT size efficient technique to generate real time optical OFDM signals compatible with IM/DD systems," in Proc. European Microwave Conference (EuMC), pp. 1247-1250, Nuremberg, Germany, October 2013. 